\title{
Combination of cetuximab and PP242 synergistically suppress the progression of wild-type KRAS colorectal carcinoma
}

\author{
Lei Cheng',* \\ Zuguang $\mathrm{Xia}^{2,3, *}$ \\ Xinyu Bian' \\ Guangchao $\mathrm{Li}^{4}$ \\ Jing $\mathrm{Hu}^{\prime}$ \\ $\mathrm{Ya} \mathrm{CaO}^{5}$ \\ Qing Wang' \\ Xiaoping Qian'
}

'Comprehensive Cancer Center of Drum-Tower Hospital, Medical School of Nanjing University,

Clinical Cancer Institute of Nanjing

University, Nanjing, ${ }^{2}$ Department of

Medical Oncology, Fudan University

Shanghai Cancer Center, Shanghai,

${ }^{3}$ Department of Oncology, Shanghai

Medical College, Fudan University,

Shanghai, ${ }^{4}$ School of Bioscience

and Bioengineering, South China

University of Technology, Guangzhou,

Guangdong, ${ }^{5}$ Department of Biological

Science and Technology and State

Key Laboratory of Pharmaceutical

Biotechnology, School of Life Sciences,

Nanjing University, Nanjing, People's

Republic of China

*These authors contributed equally to this work

This article was published in the following Dove Press journal:

OncoTargets and Therapy

2 November 2015

Number of times this article has been viewed

\begin{abstract}
Mammalian target of rapamycin (mTOR) has been shown to be overactive in human colorectal cancer, but the first-generation mTOR inhibitor, rapamycin, has failed to show clinical efficacy against colorectal cancer. On the other hand, although the secondgeneration mTOR inhibitor, PP242, has exerted substantial efficacy, it was revealed that independent inhibition by PP242 was transient, which could lead to positive-feedback loop to EGFR. Using wild-type KRAS colorectal cancer cells as models, we investigate the treatment efficacy of a widely used anti-EGFR monoclonal antibody, cetuximab, and PP242, alone or in combination in vitro and in vivo. Results of cell viability assays confirmed the synergistic inhibitory effect of PP242 and cetuximab on the survival of Caco-2 and HT-29 cells. Moreover, the ability of cancer-cell invasion and proliferation was also significantly inhibited by the combination therapy when compared with cetuximab or PP242 alone. Interestingly, the percentage of CD44-positive cancer cells was substantially decreased by the combination therapy in comparison with PP242 alone through fluorescence-activated cell sorting. The growth of cancer stem-like cell spheres in vitro was also maximally inhibited by combination therapy, in terms of either diameter or number. More importantly, the efficacy of combination therapy was more prominent than either drug alone in established tumor xenografts. These findings supported the potential use of combination therapy of PP242 and cetuximab against wild-type KRAS colorectal carcinomas.
\end{abstract}

Keywords: colorectal cancer, cancer stem-like cells, anti-EGFR treatment

\section{Introduction}

Colorectal cancer is the third most common cancer and also the second leading cause of cancer-related death in the USA. ${ }^{1}$ There have been great achievements in targeted therapy against metastatic colorectal cancer (mCRC) in recent years. According to many large clinical studies, ${ }^{2,3}$ anti-VEGF or anti-EGFR monoclonal antibody (MoAb) was well accepted as efficient therapy in combination with oxaliplatin or irinotecanbased chemotherapy against $\mathrm{mCRC}^{4}$ in first- or second-line settings.

Personalized prognosis ${ }^{5,6}$ and personalized targeted therapy ${ }^{7-9}$ based on innovate biomarkers have been emphasized during recent years, which might bring survival benefits to cancer patients. Two EGFR-related pathways, RAS-RAF-MAPK and PI3K-AKT-mTOR, were closely related to cell proliferation, metastasis, and development of colorectal cancer. Recently, changes in these pathways were also linked to anti-EGFR MoAb resistance. ${ }^{10}$ It was reported that mutations in KRAS, BRAF, and PIK3CA were associated with anti-EGFR MoAb resistance, and testing for these mutations was a potentially cost-effective way to improve survival in mCRC patients. ${ }^{11,12}$

Correspondence: Xiaoping Qian Comprehensive Cancer Center of Drum-Tower Hospital, Medical School of Nanjing University, Clinical Cancer Institute of Nanjing University, 32I Zhongshan Road, Nanjing 210008, People's Republic of China Email qianxiaoping2। I@hotmail.com 
Of note, the mammalian target of rapamycin (mTOR) has been shown to be overactive in a series of human cancers and is emerging as potential target for drug development. However, the inhibition of mTOR complex 1 (mTORC1) by the first-generation mTOR inhibitor rapamycin was incomplete. The increased mTOR complex 2 activity and the suppression of the negative-feedback loop to IR/IRS and PI3K following mTORC1 targeting were the major reasons for failure of mTORC1 inhibitors. ${ }^{13}$ After that, the emergence of next-generation mTOR inhibitors achieved exciting treatment efficacy against colorectal carcinomas in vitro, and it was interesting to observe the inhibited growth of colorectal cancer stem-like cells (CSCs) by the treatment of PP242 from the previous studies. ${ }^{14}$ On the other hand, although PP242 was capable of inhibiting both mTORC1 and mTOR complex 2 , the efficacy was also demonstrated to be transient, which was caused by the positive-feedback loop to EGFR. ${ }^{15}$

Collecting the results of the previous studies earlier, we hypothesized that it would be extremely effective against wild-type KRAS colorectal cancer with the adoption of combination treatment of anti-EGFR MoAb cetuximab (CTX) and next-generation mTOR inhibitor PP242.

\section{Materials and methods Cell line and targeted agents}

Caco-2 and HT-29, commonly used wild-type KRAS human colorectal cancer cell lines, were purchased from American Type Culture Collection (ATCC), Manassas, VA, USA. Second-generation mTOR inhibitor PP242 (Selleck Chemicals) was dissolved to stock dose with DMSO and stored at $-80^{\circ} \mathrm{C}$. CTX was kindly provided by EMD Millipore (Billerica, MA, USA) and was stored at $-20^{\circ} \mathrm{C}$.

\section{Cell viability assay and assessment of combination index}

Cells were seeded and cultured in 96-well plates at the density of 5,000 cells per well in $100 \mu \mathrm{L}$ of growth medium. Cells were treated or untreated with different doses of CTX and PP242 alone or in combination for 96 hours, then the plates were incubated at $37^{\circ} \mathrm{C}$ for 4 hours with 3-[4,5-dimethylthiazol-2yl]-2,5-diphenyltetrazolium bromide (MTT). Lastly, the MTT was replaced by DMSO, and the cell viability was measured at $405 \mathrm{~nm}$ wavelength with a microplate reader. The mode of interaction between CTX and PP242 could be analyzed by the Chou and Talalay method as indicated by previous studies. ${ }^{16}$ In order to assess whether the interactions between CTX and PP242 were synergistic, additive, or antagonistic, combination indexes (CIs) were calculated as follows:
$\mathrm{CI}=\left[\mathrm{C}_{\mathrm{CTX}}\right.$ (incombination), $\mathrm{X} \%$ effect $/ \mathrm{C}_{\mathrm{CTX}}$ (alone), $\mathrm{X} \%$ effect $]+\left[\mathrm{C}_{\mathrm{PP} 242}\right.$ (incombination), $\mathrm{X} \%$ effect/ $\mathrm{C}_{\mathrm{PP} 242}$ (alone), $\mathrm{X} \%$ effect]

where $\mathrm{C}$ is the drug concentration, by which cells can be inhibited at $\mathrm{X} \%$ effect. $\mathrm{CI}$ is the combination index. $\mathrm{CI}<1$ indicates synergistic effect; $\mathrm{CI}=1$ reflects addictive effect; and $\mathrm{CI}>1$ suggests antagonistic effect.

\section{Flow cytometry analysis}

For the CD44 detection assay, cells were incubated with antiCD44 phycoerythrin (eBioscience, San Diego, CA, USA) after being treated or untreated with CTX and PP242, alone or in combination. Immunoglobulin G-phycoerythrin was used as an isotype control. After that, CD44-positive cells were measured according to the manufacturer's instructions. CD44-positive cells in the control group (without any treatment) were selected by fluorescence-activated cell sorting (FACS) for the use of CSC sphere formation.

\section{Sphere formation assay}

CD44-positive cells were selected by FACS. Then, they were suspended in serum-free DMEM/F12 (Thermo Fisher Scientific, Waltham, MA, USA) supplemented with $20 \mathrm{ng} / \mathrm{mL}$ basic fibroblast growth factor (PeproTech, NJ, USA), $20 \mathrm{ng} / \mathrm{mL}$ epidermal growth factor (EGF) (PeproTech), 2\% B-27 supplement (Thermo Fisher Scientific), $20 \mathrm{mmol} / \mathrm{L}$ HEPES, 100 units/mL penicillin (Thermo Fisher Scientific), and $100 \mu \mathrm{g} / \mathrm{mL}$ streptomycin (Thermo Fisher Scientific) at a density of $10^{4}$ cells per well in ultralow attachment six-well plates (Corning Incorporated, Corning, NY, USA). Sphere formation was observed and images were captured using ZEISS observer A1.

\section{Colony formation assay}

A destiny of 600 cells per well were plated in six-well plates. The cells were treated or untreated with CTX and PP242, alone or in combination. The culture medium was replaced every 3 days with fresh medium containing PP242 and/or CTX. Medium was removed and cell colonies were stained with $0.5 \%$ crystal violet solution after 14 days.

\section{Transwell assay}

For transwell assay, cells were seeded and plated into the upper chambers of the 24-transwell Boyden chamber wells (Costar, Bedford, MA, USA). Cells that migrated into the lower surface were fixed and stained with $0.1 \%$ crystal violet after being treated or untreated with CTX and PP242, alone 
or in combination for 24 hours. The number of migrated cells in five random microscopic fields per chamber was countered under a light microscope.

\section{Mouse xenograft model and treatments}

Caco-2 cells were injected into the axillary regions of 6-week old female athymic (nu/nu) mice. All animal studies were performed in compliance with guidelines set by the Animal Care Committee at Drum Hospital, Nanjing, People's Republic of China. When tumors reached approximately 150-200 $\mathrm{mm}^{3}$, the mice were randomly assigned into four groups: the first group was treated with saline (control), the second group was treated with PP242 $(50 \mathrm{mg} / \mathrm{kg})$, the third group was treated with the CTX $20 \mathrm{mg} / \mathrm{kg}$, and the fourth group were treated with PP242 plus CTX. Treatments were given twice weekly. Tumor volumes and the body weight were measured once every day and calculated based on the following formula:

$$
\mathrm{V}=4 / 3 \times \pi \times\left(\text { length } / 2 \times[\text { width } / 2]^{2}\right)
$$

\section{Statistical analysis}

Statistical analyses were performed by GraphPad Prism version 5.01 software for Windows (GraphPad Software, Inc., La Jolla, CA, USA). Categorical variables were examined by Fisher's exact test. The differences in the means between two groups were analyzed with two-tailed unpaired Student's $t$-test and two-way analysis of variance.
Results were considered to be statistically significant at $P<0.05$.

\section{Results}

\section{PP242 and CTX did have synergetic effect on cell survival}

Caco-2 and HT-29 cells were treated with different concentrations of PP242 and CTX, alone or in combination for 24 hours. The inhibition ratio of the combination therapy was distinguished from either single agent for Caco-2 cells (PP242 plus CTX vs PP242, $P=0.013$; PP242 plus CTX vs CTX, $P<0.01)$ but was only distinguished from the PP242 group for HT-29 cells $(P<0.01)$ (Figure 1). The CI value (Table 1) according to the results of MTT assay was higher than 1 at some lower doses of PP242 and CTX (Caco-2 cells, PP242: 50-100 nM CTX: 0.01-0.1 $\mu \mathrm{g} / \mathrm{mL}$; HT-29 cells, PP242: 50 nM CTX: $0.01 \mu \mathrm{g} / \mathrm{mL}$ ), but was less than 1 across most concentrations, indicating a strong synergistic inhibitory effect on cell viability across most concentrations except some lower doses.

\section{Effect of combined CTX and PP242 on cancer invasiveness and the formation of colonies}

As seen in Figure 2A and B, the ability of colonial formation was significantly inhibited by the combination treatment (CTX: $20 \mu \mathrm{g} / \mathrm{mL}$; PP242: 1,000 nM), surpassing the inhibitory efficacy of CTX or PP242 alone. Compared
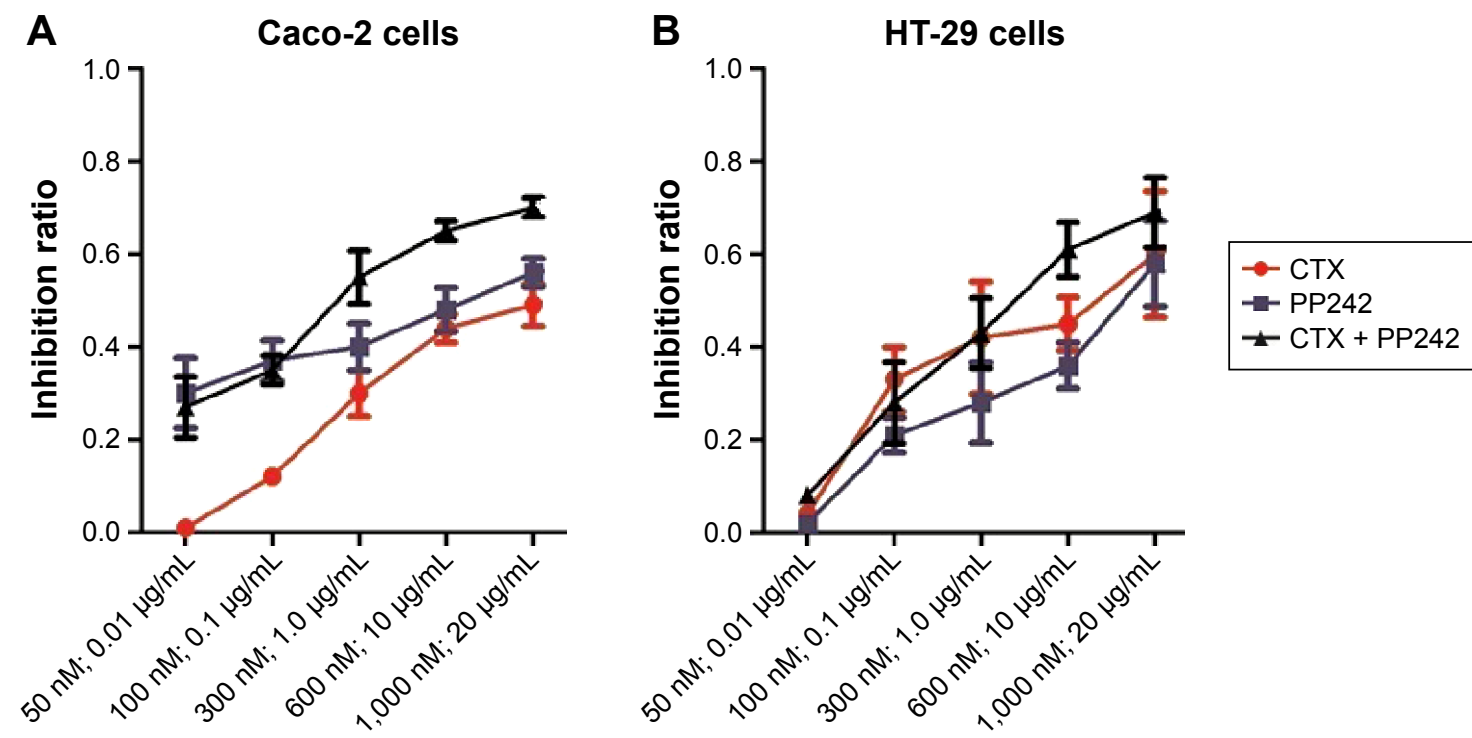

Figure I MTT analysis.

Notes: MTT analysis of (A) Caco-2 and (B) HT-29 cell inhibition ratio across different concentrations of CTX and PP242, alone or in combination. For Caco-2 cells, the efficiency of combination treatment against cell growth in vitro could be distinguished from either agent alone. However, the inhibition ratio of combination treatment was significantly higher than PP242 alone $(P=0.005)$ but not the CTX $(P=0.334)$ alone for HT-29 cells. The concentrations labeled in the $X$-axes were the corresponding concentration of PP242 $(\mathrm{nM})$ and/or CTX $(\mu \mathrm{g} / \mathrm{mL})$ in each group.

Abbreviations: CTX, cetuximab; MTT, 3-(4,5-dimethylthiazol-2-yl)-2,5-diphenyltetrazolium bromide. 
Table I Combination index of different concentrations of PP242 and CTX

\begin{tabular}{llllll}
\hline $\begin{array}{l}\text { Concentration of } \\
\text { PP242 }(\mathbf{n M})\end{array}$ & $\begin{array}{l}\text { Concentration of } \\
\text { CTX }(\mu \mathrm{g} / \mathrm{mL})\end{array}$ & Effect (Caco-2) & Effect (HT-29) & Cl (Caco-2) & Cl (HT-29) \\
\hline 50.0 & 0.01 & 0.15 & 0.08 & 1.54 & 1.80 \\
100.0 & 0.1 & 0.44 & 0.28 & 1.37 & 0.47 \\
300.0 & 1.0 & 0.60 & 0.43 & 0.30 & 0.76 \\
600.0 & 10.0 & 0.75 & 0.61 & 0.38 & 0.85 \\
$1,000.0$ & 20.0 & 0.77 & 0.69 & 0.48 & 0.87 \\
\hline
\end{tabular}

Abbreviations: $\mathrm{Cl}$, combination index; $\mathrm{CTX}$, cetuximab.

with single agent, the ability of cancer invasion was also further inhibited when PP242 and CTX was used concomitantly according to transwell assay (Figure 2C and D). Taken together, it was obvious that blocks of both EGFR and mTOR kinase activity could synergistically inhibit the proliferation and invasiveness of wild-type KRAS colorectal cancer cells.

\section{Combination of CTX and PP242 synergistically suppressed colorectal CSCs}

To determine the efficacy of the combination therapy against CSCs, we first treated adhered Caco-2 cells with PP242 and CTX, alone or in combination. The percentage of CD44-positive cells, which is a well-recognized biomarker of CSCs, ${ }^{17-19}$ was found to be significantly decreased under the treatment of the combination therapy for 24 hours compared with single PP242 and the control group according to the results of FACS (Figure $3 \mathrm{~A}$ and B). In addition, $\mathrm{CD}_{4} 4^{+} \mathrm{CSC}$ spheres that hold the stemness of tumor initiation and differentiation were harvested in approximately 2 weeks of cultivation in a serum-free medium. ${ }^{20}$ After that, to avoid the antagonistic effect of EGF on anti-EGFR MoAb, the concentration of EGF in each group was replaced with $0.2 \mu \mathrm{g} / \mathrm{mL}$ according to previous studies, ${ }^{21}$ and then the corresponding treatment was added to the medium. After 72 hours treatment, interestingly, the combination-therapy group was the only one that significantly decreased the number of spheres compared with the control group ( $P=0.046)$, but the results were not distinguished from PP242 $(P=0.07)$ or CTX alone $(P=0.11)$. Moreover, the diameter was found to be significantly decreased in the group of combination treatment compared with single agent and control groups (Figure 3C-F). Taken together, it seemed that the combination therapy of PP242 and CTX exerted the maximum efficacy against
A

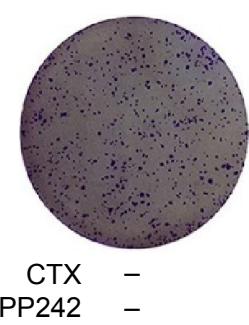

C

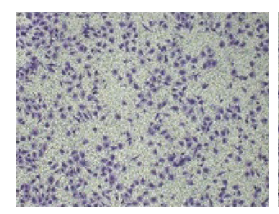

CTX -

PP242 -

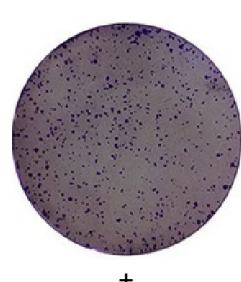

$+$

$-$

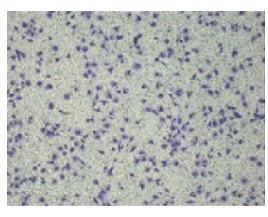

$+$

$-$

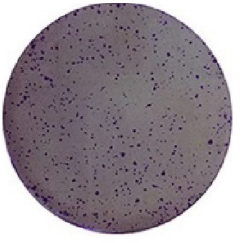

$+$

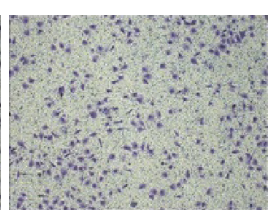

$-$
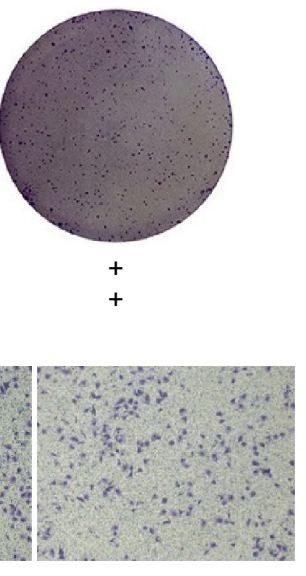

$+$

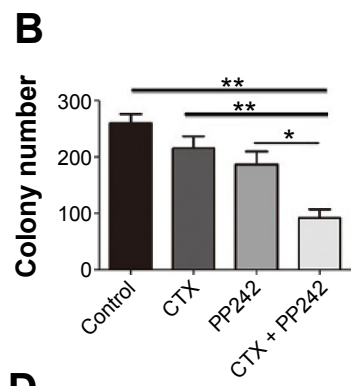

D

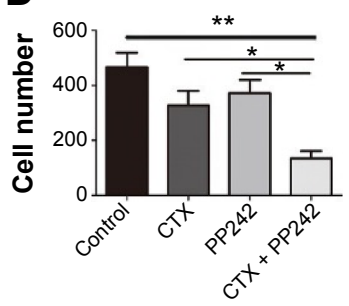

Figure 2 The synergistical efficacy of CTX plus PP242 against proliferation and invasiveness of wild-KRAS CRC cells.

Notes: (A) Colony formation assay of control group and Caco-2 cells treated with CTX and/or PP242. (B) The colony numbers in A were accounted and presented as mean + SD. The combination treatment showed the most prominent efficiency against colony formation when compared with other groups. (C) Caco-2 cells were seeded on a Matrigel-coated transwell membrane, and the treatment and analysis were similar to the colony formation assay. (D) The cell numbers invading through the membrane into the lower compartment of the chamber were counted as mean + SD.

Abbreviations: CTX, cetuximab; CRC, colorectal cancer; SD, standard deviation. 
A

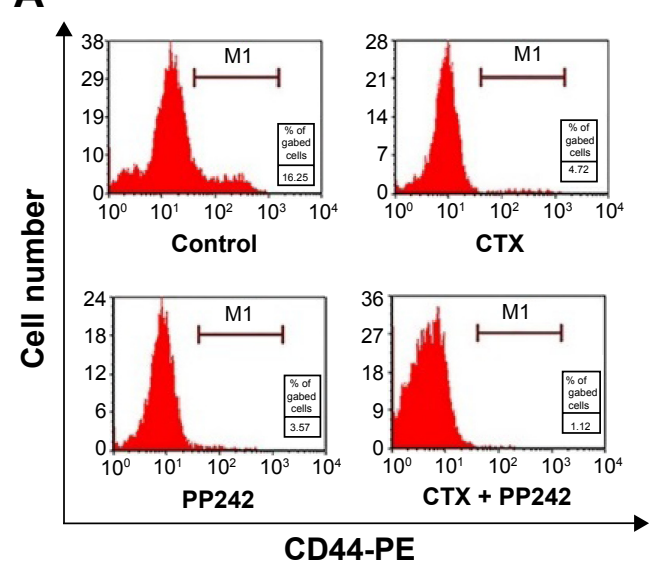

C

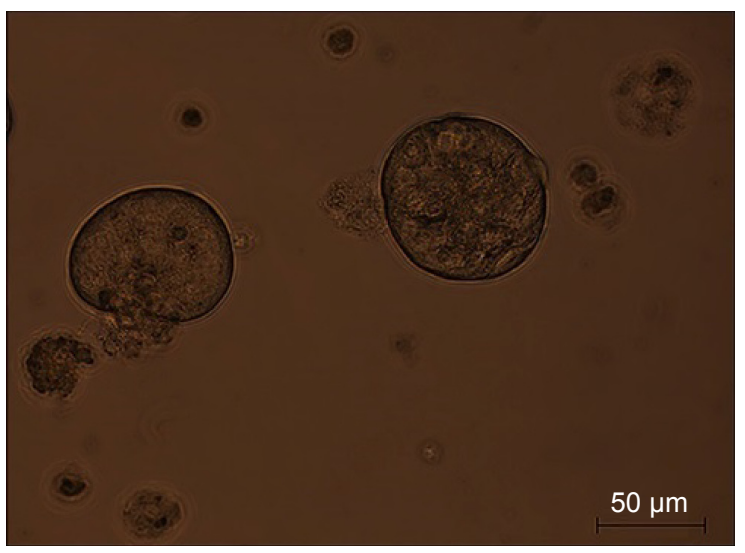

E

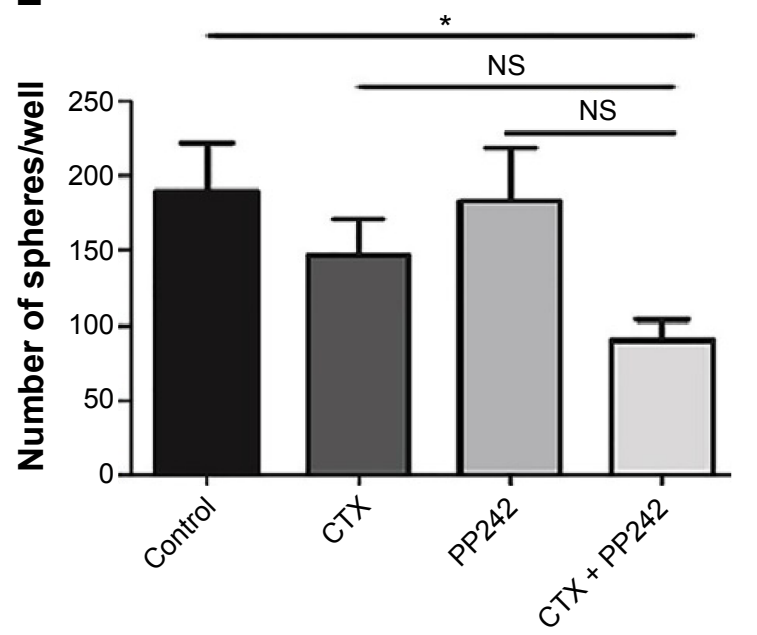

B

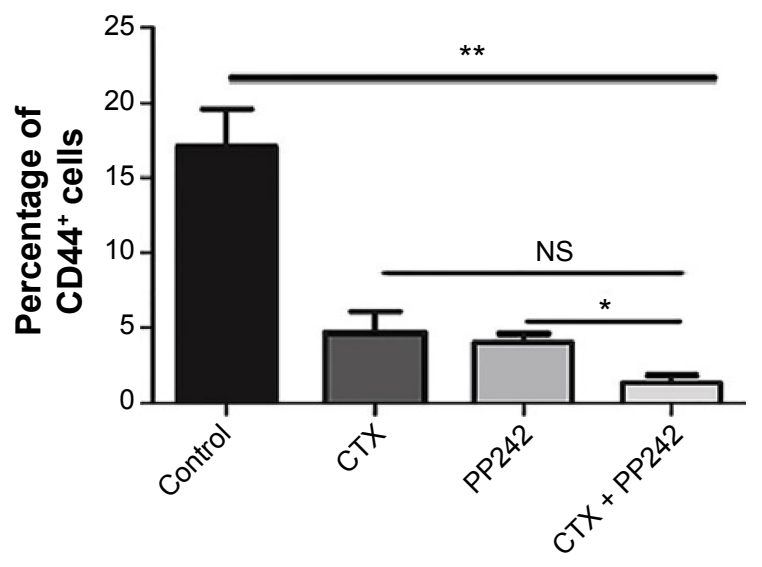

D

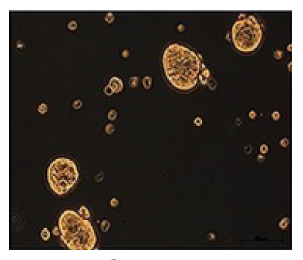

Control

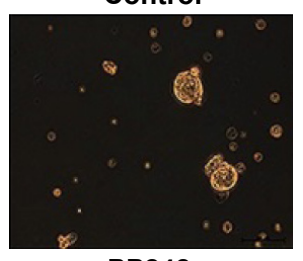

PP242

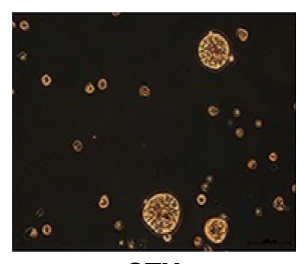

CTX

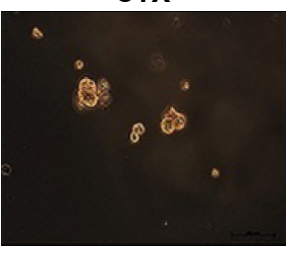

CTX + PP242

$\mathbf{F}$

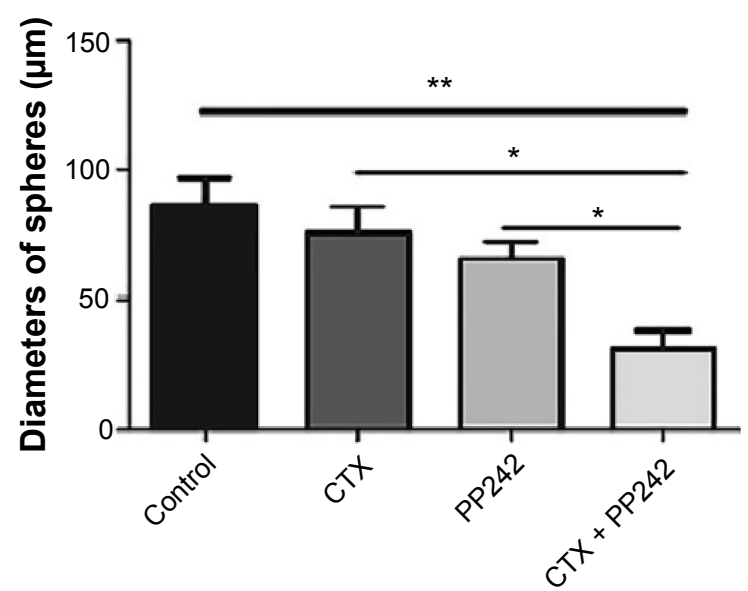

Figure 3 The synergistic efficacy of CTX plus PP242 against cancer stem-like cells.

Notes: (A) After 24 hours treatment, adhered Caco-2 cells were incubated with anti-CD44-PE and then analyzed by FACS. The combination of CTX and PP242 caused the largest reduction in the percentage of CD44+ cells. (B) Statistical analysis of the differences among the four treatment groups with regard to the percentage of CD44positive adhered Caco-2 cells. (C) CD44+ Caco-2 cells were selected by FACS and were incubated with serum-free culture mediums. This is the representative figure of cancer stem-like spheres. (D) After 72 hours treatment of different strategies, the diameter and the number of Caco-2 spheres were measured. It was obvious to observe the reduced diameter with the combinational treatment of CTX and PP242. (E and F) Statistical analysis of the differences among the four treatment groups in terms of the number and the diameters of CD44+ Caco- 2 spheres. The number of spheres was only decreased significantly in the combinational treatment group when compared with the control group, and the diameters of spheres of the combination-therapy group were significantly reduced compared with other groups after 72 hours' treatment of CTX and/or PP242. $* P<0.05 ; * * P<0.01$.

Abbreviations: CTX, cetuximab; FACS, fluorescence-activated cell sorting; NS, no significance. 
CSCs, which was potentially important for the treatment of colorectal cancer.

\section{Combination of CTX and PP242 suppresses colorectal carcinoma xenografts}

To verify the tumorigenic potential of CD44-positive CSC spheres, we first disintegrated the CSC spheres, and then injected them and the adhered cells separately into the nude mice in amounts of $10^{3}, 10^{4}$, and $10^{5}$ cells ( $\mathrm{n}=4$ per group), respectively. The tumor formation rate of mice injected with $10^{5}$ CSC sphere cells was $100 \%$ (4/4) while there were no visible tumors with the adhered cells $(0 / 4)(P=0.03)$, indicating the substantial tumorigenic attribution of CSC spheres (Table 2). To evaluate the treatment efficacy in vivo, we established the xenografts with Caco-2 cells and divided them into groups of control, PP242, CTX, and CTX plus PP242, respectively, once the tumor volume was achieved at approximately $150-200 \mathrm{~mm}^{3}$ sizes ( $\mathrm{n}=4$ per group). After 2 weeks of treatment, a significant difference in the volume of tumors was observed among the groups, and the anticancer efficacy was shown to be most prominent in the combination-therapy group. However, it was interesting to observe that there was a significant interaction between the treatment days and therapy strategies through two-way ANOVA analysis $(P<0.0001)$, and the synergistic efficacy of the combination therapy gradually increased as time went on. During the treatment progress, no significant difference was found in the body weight of the mice among all groups. Taken together, the results of the in vivo study further indicated the significant synergy of PP242 and CTX without a significant toxicity burden (Figure 4A-C).

\section{Discussion}

Personalized anti-EGFR treatment was found by several studies to be beneficial to $\mathrm{mCRC}$ patients, and was taken into clinical practice by multiple studies. ${ }^{2,22,23}$ However, primary and acquired resistance to anti-EGFR MoAb remain the major problems that restrict survival improvement. ${ }^{24,25}$ It was demonstrated that MET expression could be enhanced during the anti-EGFR treatment process, which was an important reason for acquired anti-EGFR resistance in mCRC patients. ${ }^{25}$ Of note, it was revealed that there was only limited survival improvement with the treatment of antiEGFR MoAb for wild-type KRAS mCRC patients in either the first-line (median overall survival, 23.9 vs 19.7 months, $P=0.17$ ) or second line (median overall survival, $14.5 \mathrm{vs}$ 12.5 months, $P=0.37$ ) setting by a recent study reporting the final results of the PRIME trial, ${ }^{26}$ as well as by another large prospective controlled trial. ${ }^{27}$ Finding out an antiEGFR MoAb-based combination targeted therapy which could enhance the efficacy of anti-EGFR treatment alone is potentially important.

mTOR inhibitor has also been emphasized in the treatment of colorectal cancer in recent preclinical or clinical studies. Limited stage CRC patients were mostly received chemotherapy for adjuvant therapy until the disease was progressed, and some studies confirmed that the mTOR pathway would be overactive after chemotherapy, which was a major reason for tumor recurrence. ${ }^{14}$ Moreover, it was interesting to observe the inhibition of CSC growth with the next-generation mTOR inhibitor ${ }^{13,14}$ or CTX alone, ${ }^{21}$ but it is still unknown whether there is a synergic effect against CSCs when mTOR and EGFR are co-inhibited. Given the importance of mTOR pathway in the progression and recurrence of colorectal carcinoma, and the positive-feedback loops to EGFR following therapy with next-generation mTOR inhibitor, a combination of CTX and PP242 was a reasonable strategy which might bring benefit to $\mathrm{mCRC}$ patients.

Our study is the first to demonstrate the synergistic effect of anti-EGFR MoAb and PP242 on the inhibition of the growth, invasiveness, and proliferation of wild-type KRAS $\mathrm{mCRC}$ both in vivo and in vitro. Moreover, it was interesting to recognize that the combination of these two targeted agents could synergistically inhibit the growth of CSCs. Compared with single agents, the percentages of CD44positive Caco-2-adhered cells were decreased significantly under the therapy of CTX plus PP242, and either the diameter or number of the spheroid CSCs was also decreased by the combination therapy. CSCs were demonstrated as a subset of tumors associated with the ability to give rise to all cell types found in a particular cancer sample. Given increasing evidences that CSCs play an important role in anti-tumor drug resistance, tumor initiation, tumor recurrence, metastasis, and cancer immunity, finding a promising targeted therapy against CSCs may provide clinical improvement to $\mathrm{mCRC}$ patients. Our study is the first to demonstrate the promising treatment efficacy against colorectal CSCs with the combination block of EGFR and mTOR, which was encouraging and potentially useful in clinical setting.

However, it was puzzling to observe from the results of MTT that there was a slight to medium antagonistic effect when the dose of PP242 and CTX was low (CIs >1). The synergistic effect was most prominent when the dose spectrum was near the $\mathrm{IC}_{50}$ of PP242. The results reminded 
Table 2 The oncogenicity of cancer stem-like cell spheres and adhered cells

\begin{tabular}{llll}
\hline Cell number injected & Spheres & Adhered cells & $P$-value \\
\hline $10^{3}$ & $1 / 4$ & $0 / 4$ & 1 \\
$10^{4}$ & $3 / 4$ & $0 / 4$ & 0.14 \\
$10^{5}$ & $4 / 4$ & $0 / 4$ & 0.03 \\
\hline
\end{tabular}

us that we should adapt an appropriate dose to achieve the best efficacy.

Combination target therapy for colorectal cancer was a focused issue in recent years. In this year, the addition of pertuzumab to trastuzumab and docetaxel resulted in great overall survival improvement (56.5 vs 40.8 months; hazard ratio, 0.68 ; $95 \%$ confidence interval, $0.56-0.84$ ) for HER2-positive metastatic breast cancer as compared with the addition of placebo. ${ }^{28}$ According to recent studies, of note, it was revealed a bypass of independence on original pathways through the activation of alternative RTKs. ${ }^{29}$ However, the combination of CTX and IGFR inhibitor, and CTX plus brivanib which was a dual inhibitor of VEGFR and FGFR, both resulted in unsubstantial treatment efficacy with largely damaged health-related quality of life. ${ }^{30,31}$ Everolimus, a ramification of rapamycin, was administrated in combination with bevacizumab in a recent Phase I study. ${ }^{32}$ Although modest activity against refractory $\mathrm{mCRC}$ patients was observed, it might lead to the risks of mucosal damage and/or reduced wound healing. ${ }^{32}$ For mutant-KRAS patients, although both oral everolimus and EGFR tyrosine kinase inhibitor lapatinib could significantly inhibit the colorectal tumor growth in tumor xenograft model, only a slight increase in the efficacy was observed when receiving a combination strategy. ${ }^{33}$ However, there is a lack of encouraging clinical evidences when it comes to the combinational block of EGFR and mTOR. Given the synergistic treatment efficacy of PP242 and CTX against wild-type KRAS CRC cells and the corresponding CSCs, the combination of CTX and PP242 is expected to be used for clinical trials in the future.

\section{Disclosure}

The authors report no conflicts of interest in this work.
A

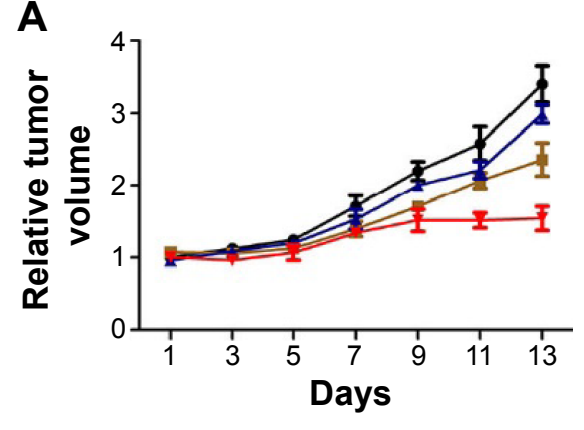

B

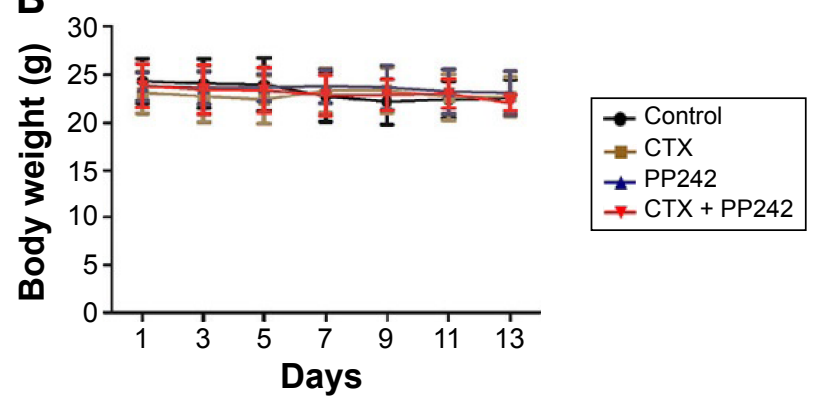

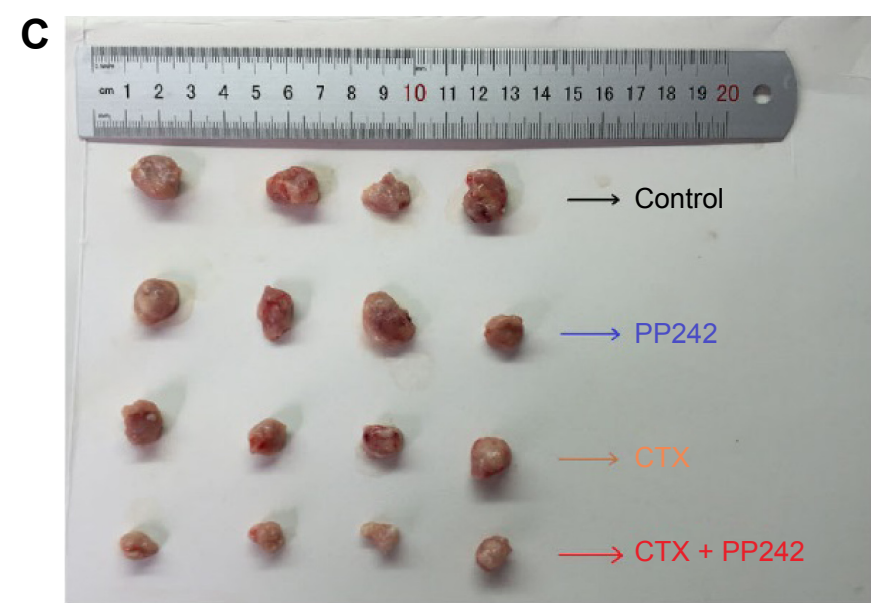

Figure 4 The efficacy of CTX plus PP242 against tumor xenografts.

Notes: (A) After inoculation of Caco-2 cells, CTX $(20 \mathrm{mg} / \mathrm{kg})$ and PP2 $242(50 \mathrm{mg} / \mathrm{kg})$ was injected into nude mice twice a week for 14 days. The body weight was measured every day, and the tumor volumes were analyzed. The treatment efficiency of CTX plus PP242 was distinguished from other groups, and the synergistic efficient was gradually increased as time went on. (B) The combination treatment had no significant effects on the body weight of mice during the treatments. (C) Representative figure of subcutaneous tumors from all groups. Tumors were most sensitive to the combination of PP242 and CTX when compared with other groups.

Abbreviation: CTX, cetuximab. 


\section{References}

1. Jemal A, Bray F, Center MM, Ferlay J, Ward E, Forman D. Global cancer statistics. CA Cancer J Clin. 2011;61(2):69-90.

2. Douillard JY, Siena S, Cassidy J, et al. Randomized, phase III trial of panitumumab with infusional fluorouracil, leucovorin, and oxaliplatin (FOLFOX4) versus FOLFOX4 alone as first-line treatment in patients with previously untreated metastatic colorectal cancer: the PRIME study. J Clin Oncol. 2010;28(31):4697-4705.

3. De Roock W, Piessevaux H, De Schutter J, et al. KRAS wild-type state predicts survival and is associated to early radiological response in metastatic colorectal cancer treated with cetuximab. Ann Oncol. 2008;19(3):508-515.

4. Hickish T, Cassidy J, Propper D, et al. A randomised, open-label phase II trial of afatinib versus cetuximab in patients with metastatic colorectal cancer. Eur J Cancer. 2014;50(18):3136-3144.

5. Tutar Y. miRNA and cancer; computational and experimental approaches. Curr Pharm Biotechnol. 2014;15(5):429.

6. Xu L, Li M, Wang M, Yan D, Feng G, An G. The expression of microRNA-375 in plasma and tissue is matched in human colorectal cancer. BMC Cancer. 2014;14:714.

7. Purohit R. Role of ELA region in auto-activation of mutant KIT receptor: a molecular dynamics simulation insight. J Biomol Struct Dyn. 2014;32(7):1033-1046.

8. Rajendran V, Sethumadhavan R. Drug resistance mechanism of PncA in Mycobacterium tuberculosis. J Biomol Struct Dyn. 2014;32(2):209-221.

9. Rajendran V, Purohit R, Sethumadhavan R. In silico investigation of molecular mechanism of laminopathy caused by a point mutation (R482W) in lamin A/C protein. Amino Acids. 2012;43(2):603-615.

10. Cheng L, Ren W, Xie L, et al. Anti-EGFR MoAb treatment in colorectal cancer: limitations, controversies, and contradictories. Cancer Chemother Pharmacol. 2014;74(1):1-13.

11. Kircher SM, Mohindra N, Nimeiri H. Cost estimates and economic implications of expanded RAS testing in metastatic colorectal cancer. Oncologist. 2015;20(1):14-18.

12. García-Foncillas J, Díaz-Rubio E. Progress in metastatic colorectal cancer: growing role of cetuximab to optimize clinical outcome. Clin Transl Oncol. 2010;12(8):533-542.

13. Francipane MG, Lagasse E. mTOR pathway in colorectal cancer: an update. Oncotarget. 2014;5(1):49-66.

14. Cai Z, Ke J, He X, et al. Significance of mTOR signaling and its inhibitor against cancer stem-like cells in colorectal cancer. Ann Surg Oncol. 2014;21(1):179-188.

15. Wang Q, Wei F, Li C, et al. Combination of mTOR and EGFR kinase inhibitors blocks mTORC1 and mTORC2 kinase activity and suppresses the progression of colorectal carcinoma. PloS One. 2013;8(8):e73175.

16. Chou TC, Talalay P. Quantitative analysis of dose-effect relationships: the combined effects of multiple drugs or enzyme inhibitors. Adv Enzyme Regul. 1984;22:27-55.

17. Katoh S, Goi T, Naruse T, et al. Cancer stem cell marker in circulating tumor cells: expression of CD44 variant exon 9 is strongly correlated to treatment refractoriness, recurrence and prognosis of human colorectal cancer. Anticancer Res. 2015;35(1):239-244.
18. Todaro M, Gaggianesi M, Catalano V, et al. CD44v6 is a marker of constitutive and reprogrammed cancer stem cells driving colon cancer metastasis. Cell Stem Cell. 2014;14(3):342-356.

19. Du L, Wang H, He L, et al. CD44 is of functional importance for colorectal cancer stem cells. Clin Cancer Res. 2008;14(21):6751-6760.

20. Ricci-Vitiani L, Lombardi DG, Pilozzi E, et al. Identification and expansion of human colon-cancer-initiating cells. Nature. 2007;445(7123):111-115.

21. Luraghi P, Reato G, Cipriano E, et al. MET signaling in colon cancer stem-like cells blunts the therapeutic response to EGFR inhibitors. Cancer Res. 2014;74(6):1857-1869.

22. De Roock W, Claes B, Bernasconi D, et al. Effects of KRAS, BRAF, NRAS, and PIK3CA mutations on the efficacy of cetuximab plus chemotherapy in chemotherapy-refractory metastatic colorectal cancer: a retrospective consortium analysis. Lancet Oncol. 2010;11(8):753-762.

23. Tejpar S, Celik I, Schlichting M, Sartorius U, Bokemeyer C, Van Cutsem E. Association of KRAS G13D tumor mutations with outcome in patients with metastatic colorectal cancer treated with first-line chemotherapy with or without cetuximab. J Clin Oncol. 2012;30(29):3570-3577.

24. Cappuzzo F, Varella-Garcia M, Finocchiaro G, et al. Primary resistance to cetuximab therapy in EGFR FISH-positive colorectal cancer patients. Br J Cancer. 2008;99(1):83-89.

25. Bardelli A, Corso S, Bertotti A, et al. Amplification of the MET receptor drives resistance to anti-EGFR therapies in colorectal cancer. Cancer Discov. 2013;3(6):658-673.

26. Douillard JY, Siena S, Cassidy J, et al. Final results from PRIME: randomized phase III study of panitumumab with FOLFOX4 for first-line treatment of metastatic colorectal cancer. Ann Oncol. 2014;25(7):1346-1355.

27. Peeters M, Price TJ, Cervantes A, et al. Final results from a randomized phase 3 study of FOLFIRI $\{+/-\}$ panitumumab for second-line treatment of metastatic colorectal cancer. Ann Oncol. 2014;25(1):107-116.

28. Swain SM, Baselga J, Kim SB, et al. Pertuzumab, trastuzumab, and docetaxel in HER2-positive metastatic breast cancer. $N$ Engl J Med. 2015;372(8):724-734.

29. Harbinski F, Craig VJ, Sanghavi S, et al. Rescue screens with secreted proteins reveal compensatory potential of receptor tyrosine kinases in driving cancer growth. Cancer Discov. 2012;2(10):948-959.

30. Reidy DL, Vakiani E, Fakih MG, et al. Randomized, phase II study of the insulin-like growth factor-1 receptor inhibitor IMC-A12, with or without cetuximab, in patients with cetuximab- or panitumumab-refractory metastatic colorectal cancer. J Clin Oncol. 2010;28(27):4240-4246.

31. Siu LL, Shapiro JD, Jonker DJ, et al. Phase III randomized, placebocontrolled study of cetuximab plus brivanib alaninate versus cetuximab plus placebo in patients with metastatic, chemotherapy-refractory, wildtype K-RAS colorectal carcinoma: the NCIC Clinical Trials Group and AGITG CO.20 Trial. J Clin Oncol. 2013;31(19):2477-2484.

32. Altomare I, Bendell JC, Bullock KE, et al. A phase II trial of bevacizumab plus everolimus for patients with refractory metastatic colorectal cancer. Oncologist. 2011;16(8):1131-1137.

33. Chu C, Noël-Hudson MS, Boige V, et al. Therapeutic efficiency of everolimus and lapatinib in xenograft model of human colorectal carcinoma with KRAS mutation. Fundam Clin Pharmacol. 2013;27(4):434-442.
OncoTargets and Therapy

\section{Publish your work in this journal}

OncoTargets and Therapy is an international, peer-reviewed, open access journal focusing on the pathological basis of all cancers, potential targets for therapy and treatment protocols employed to improve the management of cancer patients. The journal also focuses on the impact of management programs and new therapeutic agents and protocols on

Submit your manuscript here: http://www.dovepress.com/oncotargets-and-therapy-journal

\section{Dovepress}

patient perspectives such as quality of life, adherence and satisfaction The manuscript management system is completely online and includes a very quick and fair peer-review system, which is all easy to use. Visit http://www.dovepress.com/testimonials.php to read real quotes from published authors. 